\title{
Effects of functional electrical stimulation (FES) on evoked muscular output in paraplegic quadriceps muscle
}

\author{
E Rabischong $\mathrm{PhD},{ }^{1,2} \mathrm{~F}$ Ohanna $\mathrm{MD}^{2}$ \\ ${ }^{1}$ Inserm U 103, Appareil Moteur et Handicap, 395 Avenue des Moulins, 34080 \\ Montpellier; ${ }^{2}$ Centre Propara, 263 rue du Caducée, Parc Euromédecine, 34080 \\ Montpellier, France.
}

\begin{abstract}
In order to assess the effects of FES on muscle output, chronic electrical stimulation of the quadriceps muscle was applied for half an hour twice a day for 2 months, in 10 thoracic level traumatic paraplegic patients. Results concerning torque (at 6 different muscle lengths) and fatigue were measured using a strain gauge transducer in isometric condition, and compared with the findings in 15 paraplegic patients who had not received electrical stimulation, and with 10 able bodied subjects with normal motor functions. With training, muscle strength was very significantly improved whilst fatigue resistance remained at a low level. The peak torque was not found to be of the same muscle length when comparing paraplegics and control subjects; it seemed to demonstrate that length-tension relationship of the muscular actuator was changing when it was electrically activated. Moreover, the force recorded in paraplegics remained markedly lower than in able bodied people.
\end{abstract}

Key words: functional electrical stimulation; muscle fatigue; muscle torque; paraplegics; quadriceps muscle.

\section{Introduction}

The main interest of functional electrical stimulation (FES) lies in the possibility to stimulate, and even to control sublesional muscles in paraplegics. Integrity of the second motoneurone is necessary. The evolution toward closed loop control permits better knowledge of muscle output using FES. At this level the problem lies in the actuator itself which is highly non linear, ${ }^{1,2}$ considering the steep slope of its recruitment curve, and whose output is time varying. The torques required for functional activities are relatively high, ${ }^{3}$ but low values could be increased with the training. ${ }^{4-6}$.

It appeared important to know the effects of FES on paraplegic muscles and compare them with normal output. Most of the models and control systems are designed for near normal performances. Variation of the output with muscle length and with time are important features in the control design.

\section{Methods}

\section{Population}

The assessment of force and fatigue was performed on paraplegic patients and on non paraplegic people. The paraplegic population $(n=25$, male $=22$, female $=3)$ was divided into 2 groups: 15 patients (group I, male $=12$, female $=3$ ) whose muscle performances were only assessed without any special training; and a second group of 10 patients (group II, male $=10$ ) whose quadriceps muscle was trained for half an hour twice a day for 2 months and then assessed. This training was performed with the patients lying down. Muscle movement was studied with the knee flexed at $30^{\circ}$, in isometric condition, without any added load. The ratio on/off was $1 / 3$ the first month $(5 / 15 \mathrm{sec})$ and $1 / 2$ the second $(6 / 12 \mathrm{sec})$. All of the spinal cord injured patients had complete lesions, extending from $\mathrm{T} 2$ to $\mathrm{T} 10$, and were studied during 
their rehabilitation. The mean age was 27 years. No patient had severe spasticity or spasms and the quadriceps reflex was present in each patient. The time after the injury was in the main between 6 months and 12 months (92\%), with a maximum of 6 years $(8 \%)$. At the end of the 2 months of training, the results were compared between groups I, II, and III. The latter was composed of 10 healthy subjects (male $=6$, female $=4$ ), with no regular sporting involvement and with a mean age of 25 years.

\section{Current generator and transducer}

For the training and the tests a commercially available 2-channel current regulated generator (Stipro 10, Selectron) was used. Pulse width was adjusted at $0.3 \mathrm{~ms}$, with a frequency of $20 \mathrm{~Hz}$, which corresponds to the most common parameters used for FES. The maximum output of this generator was $100 \mathrm{~mA}$. The electrodes used for the measurements and the training were also commercially available (3M, ref 6225 , $50 \times 50 \mathrm{~mm})$, reusable 10 times. When we were recording data a new set of electrodes was used each time. The indifferent electrode was placed just above the patella, and the cathode on the vastus lateralis, $25 \mathrm{~cm}$ above and laterally. This placement was retained after several force tests and cor- responded to the best performance recorded; with the size of the electrical field it was possible to stimulate the different parts of the muscle via their nerve.

To record the data, we have designed a force transducer with strain gauges (Wheatstone bridge) placed in such a way that only axial efforts were measured. It was mounted on an axis enabling us to adjust it on the anterior aspect of the leg. It was padded with stuffing to avoid polysynaptic withdrawal reflexes from pain. The bridge was supplied $( \pm 5 \mathrm{~V})$ by a plotter Sensormedics (Dynagraph Recorder R 611), with a $30 \mathrm{~Hz}$ filtering. The sensitivity was $0.1 \mathrm{mV} / \mathrm{mm}$ for the patients and $0.5 \mathrm{mV} / \mathrm{mm}$ for the check sample of normal subjects. The transducer was calibrated before each set of measurements $(2.5 \mathrm{~N} / \mathrm{mm}$ for group I and II, and $10 \mathrm{~N} / \mathrm{mm}$ for group III). The subject sat in a special chair with the hip flexed at $90^{\circ}$, and the thigh fixed to the chair (Fig 1).

\section{Force measurements}

An arm placed lateral to the knee and fixed on the chair has permitted a study of different muscle lengths for $90^{\circ}, 70^{\circ}, 60^{\circ}$, $45^{\circ}, 25^{\circ}$ and $0^{\circ}$ of knee flexion. The train pulses, with maximum current output, were applied for 2 seconds in order to reach a maximum tension output $(0.5 \mathrm{~s}$ to establish

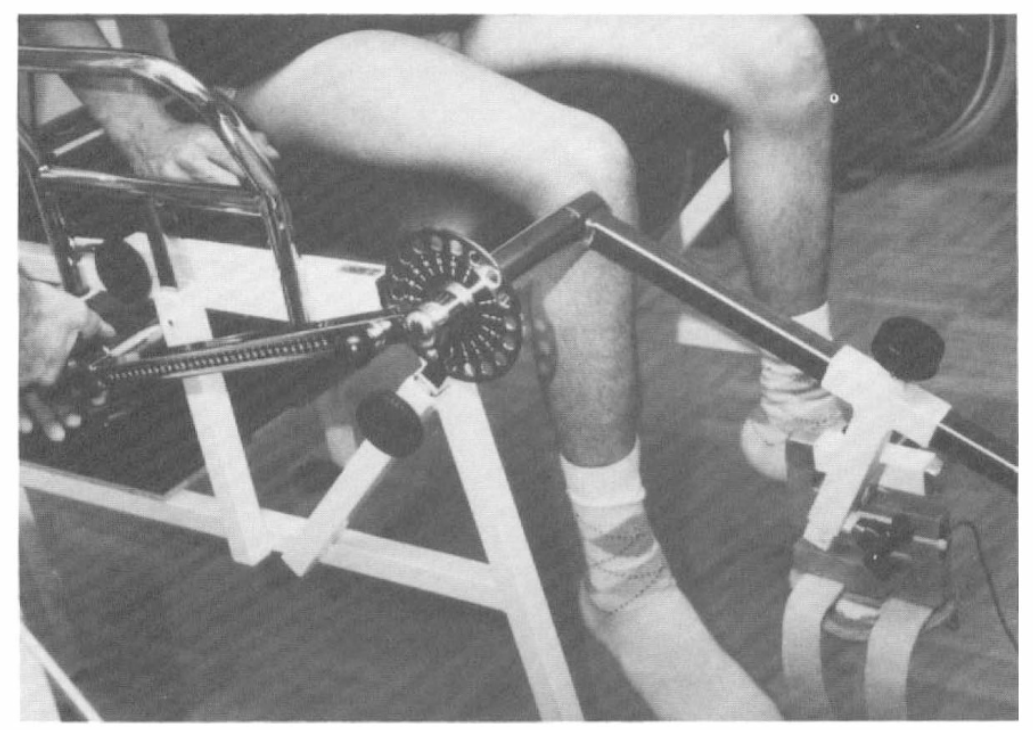

Figure 1 Device used for the assessment. 
maximum pulse amplitude), with a 30 seconds rest between each position assessed. The device described provided a constant lever of the torque, which simplified calculation. For the third group the test was performed with the same device but with voluntary muscle contraction.

\section{Fatigue measurements}

Fatigue represents force variation as a function of time. We have used a continuous train of pulses and recorded the force variation with the transducer. This continuous stimulation amplified the fatigue phenomenon but it was, nevertheless, closer to the actual use of FES for paraplegic patients where muscle activation lasts for a long period of time. The test was performed 15 minutes after the force test during the same session. The subjects in the third group were asked to exert maximum effort against the transducer for a period of 90 seconds.

Fatigue indexes (FI), expressed as percentage of force decay, were calculated each 30 seconds and for 90 seconds: $\mathrm{FI}_{\mathrm{i}}=$ $\left.\left(\left(\mathrm{X}_{0}-\mathrm{X}_{\mathrm{i}}\right) / \mathrm{X}_{0}\right)\right) 100$, where $\mathrm{X}_{0}$ represents the initial tension developed by the quadriceps with or without stimulation, $X_{i}$ the tension measured $X$ after i seconds $(30,60$ and 90). All the tests were performed in the position of the maximal torque that was recorded.

\section{Results}

The normality of distribution and the equality of variance were not confirmed in our small samples. Therefore, we have used the Mann-Withney non parametric test, which compared the sample values, using their rank of observation. Differences were considered significant when $p<0.05$, We have studied absolute values, ie values expressed with a force or torque dimension, and relative values, ie values expressed as percentage of maximal values.

\section{Torque}

Table I shows the average value of absolute torque reached by each group at different muscle length (Fig 2). Group III exhibited the best performances. The maximum torque was on average $113.16 \mathrm{Nm}$ at $90^{\circ}$ of knee flexion. The linear regression with torque expressed as a function of muscle length showed high values of correlation:

$$
\begin{aligned}
\text { Absolute torque }= & 1.008(\text { knee position }) \\
& +27.17,
\end{aligned}
$$

with $\mathrm{r}=0.987, \mathrm{r}^{2}=97.54 \%$ and $p=0.0002$.

In each subject, one of the quadriceps muscles recorded was found to be stronger than the other one in accordance with the dominant lower limb. Even in this group of patients, standard deviation was high, explaining that the performances were not homogeneous between subjects.

For group I, the absolute values were lower. The mean maximal torque was $25.7 \mathrm{Nm}$, which represented only $22.7 \%$ of the value of group III. The curve of mean torque showed 2 slopes of different sign and differences between quadriceps and between subjects were often of high values.

For group II, trained for 2 months, the values were higher than for group I. The mean maximal torque was $42.2 \mathrm{Nm}$, representing $163.7 \%$ of the maximum value of group I but only $37.9 \%$ of group III values. Disparity between subject were also quite high.

It is important to notice that minimal values for all the subjects of the 3 groups were recorded at $0^{\circ}$, where muscle was at its shortest length.

Those absolute values of torque have permitted a clear distinction between the 3 groups. When comparisons were made between groups, the levels of significance were extremely high between group I/II and group III $\left(p<4.10^{-6}\right)$ explaining important differences between able bodied subjects and paraplegics whether trained or not. Between the 2 groups of patients the $p$ values were extremely low at $60^{\circ}, 45^{\circ}$ and $25^{\circ}(p<0.0006)$ and reasonably significant at $0^{\circ}(p=0.03)$. Therefore, the 2 months training have had a significant effect on torque output in the patients trained whose performances were undoubtedly higher.

The study of how this torque was produced, ie relative values, was also of great interest (Table I, Fig 3).

For group III, maximal relative values 
Table I Absolute and relative torque developed by the 3 groups as a function of knee position (degree). Absolute torque is expressed in $\mathrm{Nm}$, and relative torque as percentage of maximum values. SD is noted for standard deviation, Min for minimum values and Max for maximum values.

\begin{tabular}{llcccccc}
\hline Absolute torque Nm & Knee Position & $90^{\circ}$ & $70^{\circ}$ & $60^{\circ}$ & $45^{\circ}$ & $25^{\circ}$ & $0^{\circ}$ \\
\hline Group I & Average & 15.59 & 22.03 & 25.78 & 20.63 & 12.76 & 5.94 \\
& SD & 8.04 & 10.79 & 11.15 & 8.16 & 6.12 & 3.72 \\
Group II & Min & 2.5 & 6.2 & 4.5 & 4.5 & 3 & 1 \\
& Max & 37.95 & 44.8 & 49.5 & 36 & 30 & 18 \\
& Average & 22.46 & 30.67 & 42.21 & 35.67 & 19.25 & 8.29 \\
& SD & 14.34 & 17.4 & 11.73 & 8.27 & 5.9 & 4.15 \\
Group III & Min & 3.1 & 8.4 & 18.6 & 20.15 & 11.2 & 2.3 \\
& Max & 57 & 72 & 59 & 51.75 & 30 & 18 \\
& Average & 113.16 & 105.46 & 90.36 & 69.69 & 46.98 & 29.94 \\
Relative torque $\%$ & SD & 26.18 & 26.94 & 23.48 & 17.35 & 12.16 & 11.81 \\
Group I & Min & 48.6 & 45.6 & 34.8 & 43.9 & 23.4 & 6.8 \\
& Max & 156 & 148.2 & 144.3 & 105.3 & 69.1 & 43.7 \\
& & & & & & & \\
Group II & Average & 55.1 & 77.7 & 98.7 & 76.6 & 49.5 & 23.8 \\
& SD & 17.3 & 15.4 & 11.2 & 19.5 & 20.5 & 14 \\
& Min & 18.1 & 40 & 60 & 41.1 & 13.3 & 3.3 \\
& Max & 100 & 100 & 100 & 100 & 100 & 59.5 \\
Group III & Average & 44.94 & 62.45 & 90.93 & 79.44 & 44.7 & 18.7 \\
& SD & 18.42 & 18.8 & 10.88 & 20.96 & 17.31 & 10.87 \\
& Min & 15.3 & 35.3 & 65.4 & 35.4 & 20.8 & 5.9 \\
& Max & 85 & 100 & 100 & 100 & 82.9 & 51 \\
& Average & 96.5 & 90.1 & 77.1 & 60.16 & 40.5 & 25.4 \\
& SD & 7.63 & 11.27 & 11.16 & 9.72 & 8.31 & 9.36 \\
& Min & 74.7 & 53.6 & 54.2 & 45 & 27.1 & 9.9 \\
& Max & 100 & 100 & 100 & 79.3 & 58.6 & 41.4 \\
\hline
\end{tabular}

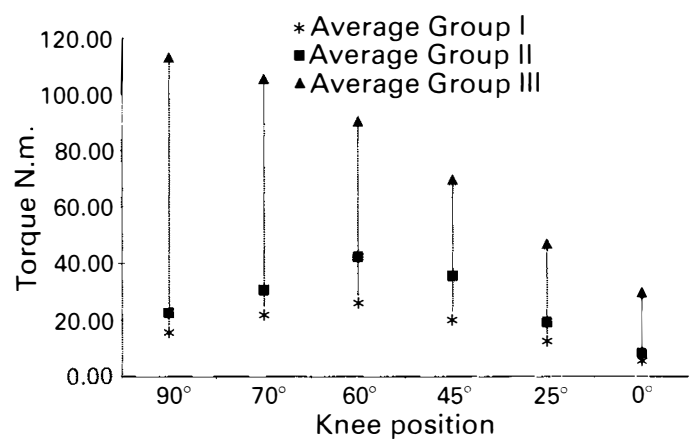

Figure 2 Absolute torque $(\mathrm{Nm})$ as a function of knee position for 3 groups.

were recorded at $90^{\circ}$ with $96.5 \%$ of maximal torque developed. Values were then decreased to a minimal at $0^{\circ}$. Standard deviation was fairly low even if absolute values exhibited important differences between subjects. Here again, regression analysis

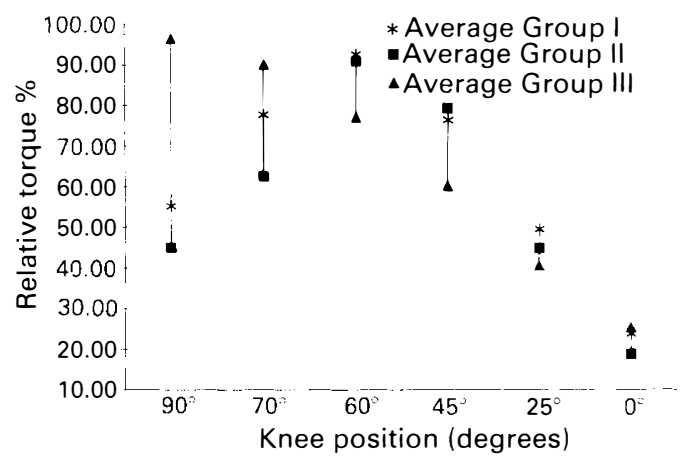

Figure 3 Relative torque, expressed as percentage of maximum values, as a function of knee position for the 3 groups.

showed high values of correlation:

$$
\begin{aligned}
\text { Relative torque }= & 0.86(\text { knee position }) \\
& +23.39
\end{aligned}
$$

with $r=0.988, r^{2}=97.67 \%$ and $p=0.0002$. 
For groups I and II maximal values were recorded at $60^{\circ}$ with, respectively, $98.7 \%$ and $90.9 \%$ of the maximum torque expressed. Minimum values were recorded at $0^{\circ}$.

The analysis indicated no significant difference between the 2 groups of patients at $60^{\circ}$, where maximum values were recorded $(p=0.4)$, while it indicated extremely significant differences between patients non trained/trained and able bodied subjects (respectively $p=7.10^{-5}, p=0.0001$ ). Patients, whether trained or not, also had differences in their torque at $70^{\circ}$ and $90^{\circ}$ $(p=0.002, p=0.02)$. At $90^{\circ}$, where maximum torque output was recorded for group III, values were extremely different between this group and the patients $\left(p<2 \cdot 10^{-7}\right)$. The differences: able bodied/ patients were maintained between $45^{\circ}$ and $90^{\circ}(p<0.002)$. Conversely, values obtained in the 3 different groups were not significantly different at $25^{\circ}$ and $0^{\circ}$ $(p>0.11)$, where muscle length were minimal.

Therefore, torque output was not only very different, considering the absolute values, between patients and valids, but also the method of expression was not comparable with a peak torque moved to $60^{\circ}$ in paraplegics.

\section{Fatigue}

As expected fatigue was a decreasing function of force with time in all the 3 groups. For groups I and II, all of the curves (Fig 4) showed comparable shapes. Force decay could be divided into 3 parts: the first one, labelled MO (maximum output), with a short plateau; the second part, labelled VO

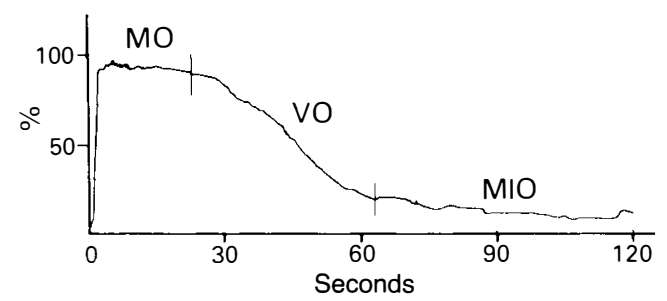

Figure 4 Typical result of fatigue assessment for the patients. MO, VO and MIO are explained in the text. (variable output); and the third one, labelled MIO (minimum output), represented a second plateau maintained for a long period of time.

The curves were, in most of the cases, smooth showing absence of perturbations induced by the stimulation. On the contrary, for group III (Fig 5) curves showed a marked oscillation showing output variation. The 3 parts were not recognised for that group.

After 30 seconds, the torque decreased by $16.2 \%( \pm 8.4)$ for group III, while it decreased by $16.1 \%( \pm 9.8)$ for group I, and $13 \%( \pm 11.9)$ for group II. After 60 seconds, the torque decreased by $27.6 \%( \pm 12.5)$ for group III, $51.7 \%( \pm 19.4)$ for group I, and $45.8 \%( \pm 16)$ for group II. After 90 seconds, torque decreased by $38.1 \%( \pm 16.7)$ for group III, $66 \%( \pm 20.5)$ for group I, and $62.6 \%( \pm 15.8)$ for group II.

FI differences between patients and control subjects were extremely significant after 30 seconds, ie for 60 and 90 seconds (respectively $p=4.10^{-5}$ and $7.10^{-5}$ for group I, and $p=6.10^{-4}$ and $1.10^{-4}$ for group II). No significant differences were calculated between patients and normal subjects for 30 seconds and between patients at all the different times $(p>0.26)$.

\section{Discussion}

Muscle output can be considered as a position dependent function in all of the 3 groups. Nevertheless, these functions did not have the same way of expression. As a matter of fact, muscle behaviour for group I and II were similar concerning the length of the muscle when maximum torque was recorded $\left(60^{\circ}\right)$, while maximum torque

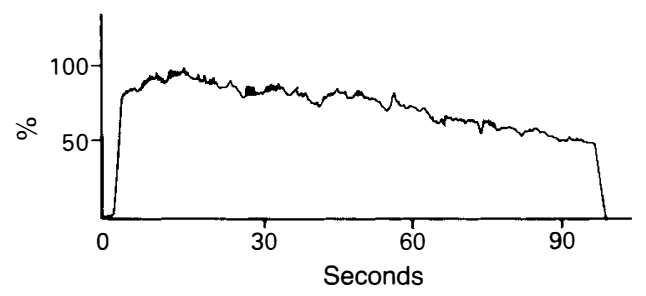

Figure 5 Typical result of fatigue assessment for group III. 
values were reached at $90^{\circ}$ for the third group. Other authors mentioned different values for able bodied subjects; eg $60^{\circ 7}$ or $75^{\circ} .{ }^{8}$ Our protocol and testing device were the same for all of the groups. Moreover, control subjects were asked to cross their arms in front of their trunk in order to avoid the possible effects of hand grasping on the measurements. Thus, we assume the homogeneousness of our protocol and measurements. Peak torque value for the paraplegic groups was recorded at $60^{\circ}$. This result was accorded to available data. ${ }^{2}$ Thus, muscle deprived from voluntary control behaved differently from muscle physiologically activated. The length-tension relationship is, physiologically, optimal at middle muscle range of motion but could be modified when the muscle is electrically activated.

Concerning output amplitude, account must be taken of the motivation of the subject coming from group III, or more generally from able bodied subjects. Performances of normal subjects of others studies were significantly higher ${ }^{7-9}$. For the trained patients, the differences of values obtained after 2 months of non time-consuming training were highly significant when compared with non trained patients. In spite of this, the performances reached stayed remarkably low compared to muscle under voluntary control. The amplitude of torque output in the trained paraplegics was inferior to the one of another study. ${ }^{5}$ This could be due to the duration of our training which appeared to be shorter. Some subjects from group III exhibited in the fatigue measurement an uncontrollable low frequency oscillation of important amplitude of the leg when maintaining the level of contraction. This movement may be permitted by the loosening of some mechanical part which supported the leg. This oscillation could be due to the fact that feedback pathways usually involve delay in the stiffness regulation. A correction applied too late may lead to an oscillation. When at some frequency of movement the limb exhibits a negative viscous stiffness, ${ }^{10}$ the effect of the stretch reflex is to assist the movement (resonant frequency).

Concerning fatigue, all the recorded curves coming from the patients exhibited 2 plateaus of different amplitude, separated by a slope, more or less steep.

The first plateau of short duration (MO on Figure 3), agreed with total muscle output developed by recruitment of motor units of different type. The amplitude of the output was the direct reflection of fast twitch and slow twitch motor units activity. As smaller motor units have larger input resistance, the recruitment under electrical stimultion is reversed from normal physiology, ie from large to small units. The fast fatiguable motor units (FF) represent the most important part of this output and their metabolism (anaerobic alactic) is dominant for the first 10 to 20 seconds; thereafter they stop their activity. This was confirmed ${ }^{11}$ by blocking local blood circulation without alteration of this phase. This output was, thus, the summation of the tension of all the different type of motor units recruited.

Then anaerobic lactic metabolism becomes dominant (VO on Figure 4), explaining the slope due to lower tension generated by motor units still discharging (fast resistant and slow). We may think longer training is acting on this slope. Therefore, the slope is a decreasing function of FR units recruitment plus the tension developed by slow twitch units, which remains constant.

The second plateau (MIO on Figure 4) represents tension of slow twitch units. This tension is functionless, but can be maintained for a long period of time.

Recording fatigue with a shorter time ${ }^{4}$ appeared not to be sufficiently sensitive to distinguish with enough accuracy the different types of fibres involved. Intermittent tests have been used in the literature ${ }^{6.12}$ but frequency and duty cycle were not compatible with real use of FES $(40 \mathrm{~Hz}$ for $330 \mathrm{~ms}$ every second and for 5 minutes). We have not noticed significant effect of the training on the fatigue resistance of our group of patients; as a matter of fact, the daily activity was not important compared to other studies ${ }^{5.6}$ which reported increase of fatigue resistance ${ }^{6}$ while using low frequency current $(10 \mathrm{~Hz})$ for long periods of time (one hour, 3 times daily for 6 weeks), but without noticeable effect on force output. A $10 \mathrm{~Hz}$ pattern of stimulation is recognised as mimicking the pattern of 
physiological activation of slow twitch units. ${ }^{13}$ Other authors ${ }^{14,15}$ consider the frequency to be of less importance compared to the amount of activity, which could explain the modulation of the motor unit typing with age and activity.

One hour of daily training, done by the patient himself, has lead to good performances concerning the torque output. We have not noticed true effect on fatigue resistance with the parameters retained and the duration of the training. Two of the trained patients were able to stand for 45 minutes at the end of the 2 months training.

\section{References}

1 Gruner JA, Mason CP (1989) Nonlinear muscle recruitment during intramuscular and nerve stimulation. $J$ Rehabil Res Dev 26: 1-16.

2 Levy M, Mizrahi J, Susak Z (1990) Recruitment, force and fatigue characteristics of quadriceps muscles of paraplegics isometrically activated by surface functional electrical stimulation. J Biomed Eng 12 (5): $150-156$.

3 Yamaguchi GT, Zajac FE (1990) Restoring unassisted natural gait to paraplegics via functional neuromuscular stimulation: a computer study. IEEE Trans Biomed Eng 37: 886-902.

4 Bajd T, Kralj A, Turk R, Benko H (1990) Symmetry of FES responses in the lower extremities of paraplegic patients. J Biomed Eng 12(5): 415-418.

5 Kralj A, Badj T (1989) Functional electrical stimulation: standing and walking after spinal cord injury. CRC Press, Boca Raton: 198.

6 Scott OM, Vrbova G, Hyde SA, Dubowitz V (1985) Effects of chronic low frequency electrical stimulation on normal human tibialis anterior muscle. J Neurol Neurosurg Psychiatry 48: 774-781.

7 Selkowitz D (1985) Improvement in isometric strength of the quadriceps femoris muscle after training with electrical stimulation. Phys Ther 65(2): 186-196.

8 Thorstensson A, Grimby G, Karlsson J (1976) Force-velocity relations and fiber composition in human knee extensor muscles. J Appl Physiol 40: 12-16.

9 Stalberg E, Borges O, Ericsson M, Essen-Gustavsson B, Fawcett PRW, Nordesjo LO et al (1989) The quadriceps femoris muscle in 20-70 year-old subjects: relationship between knee extension torque, electrophysiological parameters, and muscle fiber characteristics. Muscle Nerve 12: 382-389.

10 Rack PMH (1981) Limitations of somatosensory feedback in control of posture and movement. Motor control. Handbook of Physiology. American Physiological Society, Bethesda, Maryland 2: 229-256.

11 Hainaut K, Duchateau J (1989) Muscle fatigue, effects of training and disuse. Muscle Nerve 12: 660-669.

12 Burke RE, Levine DN, Tsairis P, Zajac FE (1973) Physiological types and histochemical profiles in motor units of the cat gastrocnemius. J Physiol 234: 749-765.

13 Vrbova G (1989) The concept of neuromuscular plasticity. In: Rose FC, Jones R, Vrbova G, editors. Neuromuscular stimulation: basic concepts and clinical applications. Comp Neurol Rehabil 3: 3-14.

14 Kernell D (1986) Organization and properties of spinal motoneurones and motor units. Prog Brain Res 64: 21-30.

15 Kernell D, Eerbeek O (1989) Physiological effects of different patterns of chronic stimulation on muscle properties. In: Rose FC, Jones R, Vrbova G, editors. Neuromuscular stimulation: basic concepts and clinical applications. Comp Neurol Rehabil 3: 193-202. 